\title{
DESIGN FOR POSITIVE BREAST SELF-EXAM EXPERIENCE USING MOBILE APPS
}

\author{
Karadeniz Küçük, Aylin; \\ Şener, Bahar \\ Middle East Technical University
}

\begin{abstract}
Breast cancer is one of the most common cancer types among women, accountable for approximately 2.2 million new cases and 684,996 deaths globally in 2020. There are various screening methods to detect cancer early, and experts suggest that women should perform breast self-examination (BSE) once a month. Unfortunately, most women fail to do so even if they are aware of the risks and the importance of screening methods. The aim of this paper is to understand women's current behavior and experience with BSE and mobile BSE apps and to suggest several design dimensions for positive mobile BSE app experience by benefiting from positive technology. With this aim, three selected mobile BSE apps were used by a total of 24 participants for four weeks. A three-phase study was carried out to uncover women's pre- during-, and post-usage experiences of BSE apps. The analysis revealed six headings under two themes: strategies, limitations, and emotions under BSE theme; and the needs, limitations, and benefits under Mobile Apps theme. Consequently, based on the findings, suggestions for design dimensions for BSE apps to better meet women's needs by benefiting from levels of positive technology are made.
\end{abstract}

Keywords: Experience design, Technology, User centred design, Breast Self-Exam, BSE Apps

\section{Contact:}

Karadeniz Küçük, Aylin

Middle East Technical University

Industrial Design

Turkey

aylinkaradeniz7@gmail.com

Cite this article: Karadeniz Küçük, A., Şener, B. (2021) 'Design for Positive Breast Self-Exam Experience Using Mobile Apps', in Proceedings of the International Conference on Engineering Design (ICED21), Gothenburg, Sweden, 16-20 August 2021. DOI:10.1017/pds.2021.101 


\section{INTRODUCTION}

Breast cancer is one of the most common cancer types among women, accountable for approximately 2.2 million new cases and 684,996 deaths globally in 2020 (IARC, 2020). As early diagnosis is critical to survival, every woman should be made aware of what they need to do to check themselves. The advice might differ across different countries; however, in [anonymised] country, where this study is carried out, the [anonymised country] Ministry of Health recommends performing Breast Self-Exam (BSE) monthly, attending Clinical Breast Exam (CBE) yearly, and mammography screening every two years to women over 40 years old. BSE has a significant impact on increasing breast cancer awareness in women under the age of 40 (Reference anonymised, n.d.). On the other hand, despite most women acknowledge the possibility of developing breast cancer in their life, they do not perform BSE mostly because of fear of finding a symptom (Yang et al., 2010). In the last decade, there has been studies to positively influence user behaviour and to motivate women to carry out BSE through design interventions (e.g., Fogg, 2009; Lilley 2009; Lockton, Harrison and Stanton, 2010) and through the integration of technologies. For example, mobile apps developed for assisting women to maintain breast health with their features supporting breast self-exam, creating breast cancer awareness, offering peer support, and accompanying patients during their treatment (Bender et al., 2013). Even though there are studies that explored women's breast health behaviour and barriers, as well as the effects of mobile apps on their health behaviour change (Kissal and Beşer, 2011; McKay et al., 2018; Özkan and Taylan, 2020; Yang et al., 2010), very little research has been carried out on the relation of breast health behaviour and related mobile apps (Ribeiro, Moreira, Almeida and Santos-Silva, 2017; Ribeiro, Moreira, Almeida and Santos, 2016). This paper presents the results of a three-phase study carried out to uncover women's pre- during-, and post-usage experiences of BSE apps. The study is part of a wider research program that aims to i) uncover motivations of women to perform BSE; ii) understand women's needs to perform BSE, and iii) suggest ways to enhance women's BSE experiences through the design of mobile apps. The scope of this paper will be to understand women's current behavior and experience with BSE and BSE apps and to suggest several design dimensions for BSE apps by benefiting from positive technology. Following sections will present the overview of knowledge related to BSE, mHealth technologies, and positive technologies. After introducing the fieldwork set-up, the results and analysis will be summarised; the discussion will be provided in relation to the strategies, limitations, and emotions regarding BSE; and the needs, limitations, and benefits regarding BSE apps. Finally, the paper will conclude with suggestions for design dimensions for BSE apps to better meet women's needs by benefiting from levels of positive technology.

\section{BREAST SELF EXAM AND MOBILE BSE APPS}

Currently, there is no proven method that prevents the occurrence of breast cancer. However, prolonging patients' life expectancy with breast cancer and achieving full recovery are possible with early diagnosis with regular examination and screening. BSE, CBE, and mammography are defined as the primary screening methods recommended for the early diagnosis. BSE is not as sensitive as mammography, but it is simple, economic, safe, and a non-invasive method that does not require any special tools. Therefore, the most critical role in the early diagnosis of breast cancer falls to the person. Although the advice may differ across countries, it is commonly recommended to carry out BSE once a month using determined methods. Despite the benefits associated with BSE, several studies demonstrated that women do not perform it due to following reasons: not knowing how to perform, fear of discovering symptoms, disbelieving in their ability to perform correctly, and having low breast cancer risk perception (Al-Azmy et al., 2013; Özkan and Taylan, 2020; Yang et al., 2010). Additionally, although people may be willing to engage in healthy behaviours, they usually lack motivation to maintain it as part of their daily routine (Kaptein, De Ruyter, Markopoulos, and Aarts, 2012). As Bolier and Abello (2014) state, engagement and adaptation of healthy behaviours into daily life can increase with technology interventions. In recent years use of smartphones and mobile apps for maintaining healthcare has arisen, and such apps can create an opportunity to raise awareness to prevent cancer and particularly breast cancer. As of 2018, approximately 600 publicly breast healthcare apps were designed to give information about the disease and overall breast health, increase general awareness, and accompany during treatment; also, some of these apps focused on BSE (Houghton, Howland, and McDonald, 2019). 
In general, research efforts regarding the influence of technology on people examine the negative effects, however only a few of them investigate how technology could contribute to happiness and well-being of people (Gaggioli et al., 2017). Positive technology is a relatively new and fast-growing movement aiming to foster positive growth of individuals, groups and institutions. Positive Technology combines positive psychology and technology to enhance personal and collective life (Downey, 2015). According to Botella et al., (2012) the three objectives of Positive Technologies can be observed at hedonic; eudaimonic; and social/interpersonal levels. Hedonic level refers to using technologies to trigger positive and pleasant experiences; eudaimonic level supports individuals to reach engaging and self-actualizing experiences through technologies; and social/interpersonal level aims to improve connectedness between people. The present study also takes a positive technology approach, and the results will be discussed in relation to three abovementioned levels.

\section{FIELDWORK SET-UP}

The fieldwork aimed to explore women's technology experience for BSE and to get insights about women's BSE behaviour, and understand their experience with BSE and BSE apps. Following sections will describe the selection of BSE apps, the participants and the three-phased study carried out.

\subsection{Mobile BSE App Selection}

In order to decide which mobile apps should be included in the study, a thorough evaluation is made. To include different operating platforms, two major application stores (Apple App Store for iOS operating system and Google Play Store for Android operating system) were searched using the keyword 'breast cancer' in July 2019, the matching apps were ordered by downloads. Only the apps available in both stores were included (approx. 50 apps). Then, they were divided into categories based on data classifications proposed by Bender et al. and Giunti et al. Criteria for the exclusion of the apps were: (1) having non-English description, (2) having requirement of payment, (3) aiming at a physician-oriented target audience, and (4) having purpose except early detection such as fundraising or disease management. The features of the remaining nine apps on the list were studied in detail. Then some of them eliminated based on their inclusion of four main behaviours essential for BSE (Misovich et al., 2003): 'performing BSE', 'learning more about BSE', 'discussing BSE with friends', and 'having someone remind one to perform BSE'. The final list included three apps with features that are believed to support the four behaviour: 'Know Your Lemons', 'Breast Check Now', and 'CheckYourself' (Figure 1).
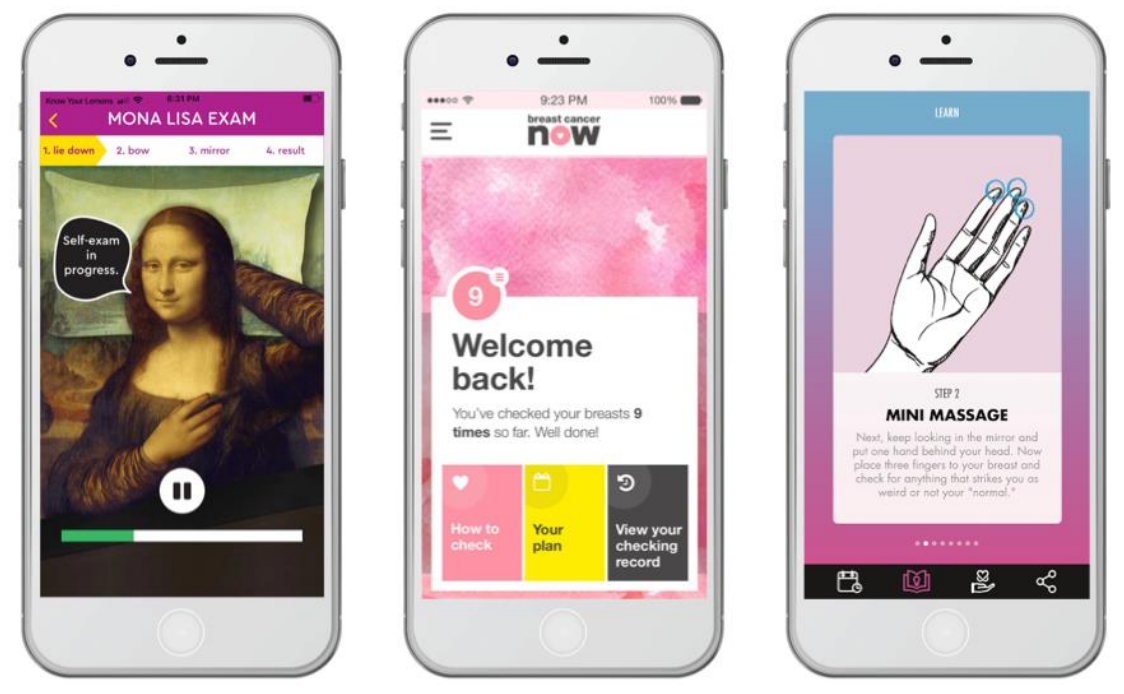

Figure 1. Screenshots from the three apps

\subsection{Participant Selection}

In order to reach to potential candidates, a web-based questionnaire is published through several social media channels (i.e., Facebook, Twitter, LinkedIn, and Instagram). The 12-item questionnaire aimed to gather general information about women's approach to BSE (e.g., their breast healthcare conditions, 
BSE knowledge), and to invite them to take part in the fieldwork. In total, 116 women completed the questionnaire, 68 of them indicated their willingness to participate. Consequently, 24 participants were selected amongst the ones who had a smart mobile phone, and an adequate level of English to be able to follow the instructions on the three selected apps. The study was conducted with 24 women, and they were divided into three groups of eight to evaluate each of the three apps. Women aged between 20 and 60 were included in the study with an average age of 35 . As much as possible, it is aimed to get homogeneous distribution between different age groups (i.e., 20-30, 30-40, and over 40 years old).

\subsection{Phases of the Fieldwork and Procedure}

The fieldwork was carried out in three-phases: i) pre-usage, ii) during usage, and iii) post-usage. All phases were carried out with each participant individually, and voice recorded. Five of the participants were interviewed face-to-face, however interviews with other participants were conducted via Skype and Zoom due to COVID-19 restrictions.

i) Pre-Usage Phase. This phase aimed to find out women's attitudes toward BSE and learn about their expectations for mobile apps. On informing participants about the study and obtaining a written consent form, the PANAS scale was administered to the participants to identify their emotions related to breast healthcare. PANAS is a widely used scale in studies that deals with mood status (Watson, Clark, \& Tellegen, 1988). Instead of mood, the term 'affect' will be used to better cover the participants' positive (e.g., enthusiasm, alertness, activity, and engagement) and negative (e.g., distress, anger, guilt, fear, and nervousness) attitudes (Mcdowell, 2006). PANAS scale required the participants to indicate on a 5-point Likert-scale (1-strongly disagree | 2-disagree | 3-Neither agree nor disagree | 4-agree | 5-strongly agree), they were also asked to fill in the Technology Readiness Index (reference to Index) to determine the level of their tendency to embrace and use new technologies. Following this, a two-part semi-structured interview was carried out with each participant. First part covered questions about participants' breast healthcare, their experiences with BSE, and mobile healthcare apps. The questions also aimed to learn about whether they performed BSE before (and if yes, their reflections). Second part of the interview concentrated on BSE apps. The participants were asked about the kinds of features they would like to/need to have in BSE apps. Subsequently, each participant was assigned one of the three apps chosen for the study. The app is introduced to the participants by using information cards describing its usage and features through a series of visuals that is prepared by the first author. Then the participants were asked to download the app, and after quick navigation, their questions (if there was) were answered. The participants then were given information about the following stages of the study. The app-feature checklist cards were also given to the participants so that they can make a note of which ones they used, their entire experience, and feelings. At this stage, each session with a participant took between 20 to 25 minutes.

ii) During-Usage Phase. In this phase, each of the eight participants were asked to use their assigned apps at least four times for a month. The authors communicated with participants via SMS to remind them to use the app and check on their experiences up to date. A few participants used the given application more than 4 times, and most participants used it four times. Some participants, who already used the app four times before the completion of a month were invited to an interview.

iii) Post-Usage Phase. In this phase, the participants were interviewed to reflect on their experiences on the app that they used. First, the post-usage PANAS scale was administered to identify the mood differences between before and after the study. Then, semi-structured interview sessions were conducted individually with each of the 24 participants. The interview contained questions about BSE experiences whilst using the app, whether the participants would like to continue performing BSE and use the given app, how the BSE apps make them feel, and their recommendations for apps. The feature checklists and their notes were also collected. Each session took between 20 to 25 minutes.

\section{FIELDWORK ANALYSIS AND RESULTS}

\subsection{Data Analysis Overview}

Analysis of the data carried out in several steps. First, an analysis carried out to evaluate pre-PANAS and post-PANAS scales administered before and after the usage of mobile BSE apps to see the changes in users attitudes. Additionally, Grounded Theory formed the basis of the analysis since understanding the participants' experiences thoroughly was critical to the study. Accordingly, 
Grounded theory's three coding types (i.e. open, axial, and selective) were applied. The researcher's notes and voice-recordings of 48 interview sessions (pre- and post-interviews with each of the 24 participants) were transcribed. As suggested by Bogdan and Biklen (2007), repetitive patterns were identified and relevant codes were assigned. It is important to note that participants were asked openended questions, and thus, not every participant may have given a response regarding that specific (assigned) code. Once obtaining initial codes, the process of thematizing was followed to create headings. Then, the codes listed under these headings were examined separately and divided into categories in accordance with the repeated patterns by applying axial coding. Then pre-categorization through axial coding was re-examined and combined under relevant sub-categories. The study had an inductive nature since there were no specific predetermined codes about women's experiences of BSE and mobile BSE apps.

\subsection{Pre- \& Post- PANAS Scales for the App Usage}

From the PANAS 'before' and 'after' data, all positive affect measures (except 'alert') increased, whilst all negative measures (except 'distressed' and 'scared') decreased. A paired t-test $(\alpha=0.05)$ was performed on the PANAS data to check for significant differences. For positive affect, comparison of the 'before' data $(\mathrm{M}=2.14, \mathrm{SD}=0.50)$ and 'after' data $(\mathrm{M}=3.36, \mathrm{SD}=0.77)$ shows a highly significant increase in positive affect, $\mathrm{t}(9)=4.70, \mathrm{p}=.001$. Similarly, for negative affect, comparison of the 'before' data $(\mathrm{M}=2.34, \mathrm{SD}=0.27)$ and 'after' data $(\mathrm{M}=1.97, \mathrm{SD}=0.42)$ shows a significant reduction in negative affect, $t(9)=3.07, p=.013$. In combination, these results confirm that the apps were successful in their task of supporting positive affect and alleviating negative affect. Figure 2 shows the mean values for 'Before' and 'After' app usages for Positive Affect (PA) and Negative Affect (NA) PANAS scales.

\begin{tabular}{lccllll}
\hline Positive Affect: PA & 'Before' & 'After' & Negative Affect: NA & 'Before' & 'After' \\
\hline Interested & 2.83 & 3.79 & Distressed & 2.16 & 2.5 \\
Excited & 2.08 & 2.87 & Upset & 1.79 & 1.37 \\
Strong & 2.33 & 4.04 & Guilty & 2.29 & 1.70 \\
Enthusiastic & 2.04 & 3.54 & Scared & 2.20 & 2.29 \\
Proud & 1.66 & 4.20 & Hostile & 2.16 & 1.62 \\
Alert & 2.79 & 2.79 & Irritable & 2.41 & 1.75 \\
Inspired & 1.37 & 3.58 & Ashamed & 2.50 & 1.46 \\
Determined & 2.37 & 3.54 & Nervous & 2.54 & 2.33 \\
Attentive & 1.50 & 1.58 & Jittery & 2.58 & 2.25 \\
Active & 2.37 & 3.62 & & Afraid & 2.75 & 2.37 \\
\hline
\end{tabular}

Figure 2. Mean value of the PANAS answers in relation to 'before' and 'after' the app usage by 24 participants

\subsection{Categories as Source for Design Strategies}

The analysis of the interview results enabled to cluster discussions for design strategies under two main topics: 1) Breast Self-Exam, and 2) Mobile App.

\subsubsection{Breast Self-Exam}

Design strategies classified under "Breast Self-Exam" theme consisted of the following headings: i) strategies, ii) limitations, and iii) emotions.

i) Strategies: This included the tactics developed to learn about BSE and to overcome difficulties related to performing BSE, which had three categories as Performing BSE, Learning BSE, and Getting help for BSE. The categories were generally related to methods that women improved for how, why, when, and where i) to perform BSE, ii) to learn BSE, and iii) to get help for BSE. For example, half of the participants (12 out of 24) emphasized that they check their breasts quickly without following any process. Additionally, 17 participants stated that they remember to perform BSE when they have sensitivity in their breasts during menstruation.

"I do breast self-exams unconsciously; they are more like a quick check. Also, my breasts become tender, especially during menstruation and it makes me anxious usually. That's why I remember performing BSE." (P09) 
Several participants indicated that they usually learned about BSE from the sources like magazines, articles on the internet, and brochures in the healthcare clinic, etc. In addition, most participants mentioned that they got help from their social network or consulted a doctor to get familiar with BSE and what to do if they find anything suspicious during BSE.

ii) Limitations: This heading covered restrictive situations that participants dealt with. It included three categories: Informational Barrier, Practical Barriers, and Psychological Barriers. Some limitations arising from practical, individual, and social restrictions related to knowledge were named as 'informational barriers'. Besides, women's concerns about performing BSE accurately, misunderstanding of the findings, and mixing the symptoms and normal breast tissue shaped the 'practical barriers to understand the BSE process and findings'. For example, a participant mentioned:

"When I performed BSE for the first time, I was unsure about whether I was doing it correctly or not. I still cannot be sure during performing. Actually, I felt a cysts in my breast and tried to understand the location and size of it. However, I am unsure about findings whenever I perform BSE." (P13)

The participants statements related to limitations caused by fear and negligence were placed under the psychological barriers like the statement of $\mathrm{P} 04$ :

"This isn't just about breast health; it can be a little scary to self-detect a general symptom. I prefer to see a doctor. On the other hand, I think that nothing will happen to me since I am healthy now."

iii) Emotions: Emotions arise from performing BSE were gathered under this category. Emotions related to Experience, Emotions related to Performing BSE, and Emotions related to Results were the three-category. All participants negative emotions such as fear, anxiety, panic, guilt, and stress whilst talking about one of these categories.

\subsubsection{Mobile Apps}

Design strategies classified under "Mobile Apps" theme consisted of the following headings: i) Needs, ii) Limitations, and iii) Benefits.

i) Needs: This included women's expectations regarding mobile app to enhance their BSE experience. The categories included: Feature, Content, Experience, Support, and Guidance. Feature-related needs were originated from four sub-categories as features related to BSE, Breast Healthcare, Women's Health, and Community. Most participants emphasized that it would be more beneficial to have an application that not only enables them to perform BSE, but also can monitor their health, especially gynaecological diseases.

"In fact, an application that gives information about the menstrual cycle, breast tissue, breastfeeding, and gynaecological diseases can also be considered. I would like to use that kind of application. It would be more useful because of being comprehensive. I would not prefer downloading an app that enables me to use it for only one issue." (P02)

The content was another critical category under the needs heading. User comments, women's stories related to BSE, critical breast health information, breast cancer symptoms, and BSE methods were the women's expectations to use the BSE apps. Also, several participants said that sharing new and informative content about breast health on the apps at specific time intervals enhanced their experiences. In general, the majority of participants expressed a desire for an experience where mobile apps are facilitative and customizable, enabling active participation and creating self-awareness. These needs were covered by 'experience-related need' category. Moreover, numerous participants stated that mobile BSE apps should support the BSE process by giving psychological or physical help and guide the users.

ii) Limitations: This included restrictions related to Content, Experience, Guidance, Features, and Privacy. Participants' statements associated with the lack of comprehensive information shaped the category of Content. Several participants commented that mobile apps did not provide summarized and essential information and visuals for breasts and BSE steps were inadequate. Limitations covered the problems about usability, user interface, and user experience. Almost half of the participants (10 out of 24) stated that they expected mobile BSE apps to offer more content and more detailed information as their knowledge about BSE increased throughout the process.

"At first, the early exams were a new experience for me since I have no awareness. I learn about the symptoms and methods. After a certain use, I was no longer a beginner thus I expected to see different information or interactions according to my level of knowledge." (P01) 
Another category mentioned by numerous women was Guidance. Some participants stated that mobile apps were insufficient in guiding for BSE process and analysing the findings. Moreover, the limitations of app-based features, such as being unable to take notes or to set a reminder were paired with Features. Finally, some participants had concerns that the app could share their personal information or watch them from the front camera during exams; and these concerns were located under Privacy.

iii) Benefits: This included the participants' impressions of the advantages of the mobile apps, and had four categories: Content, Features, Guidance, and Support. For the participants, the primary category of benefits to performing BSE with apps was the content related advantages. Several participants made reference to content-oriented benefits during the app use as:

"Getting information about breast health, cancer symptoms, and breast self-exam through an application made the process significantly easier." (P17)

Several features were other foregrounded benefits mentioned by participants. According to the women's responses, features such as setting reminders, socializing with friends through the application, creating your plan, and following their breasts positively affected their experiences. Several participants stated the importance of Guidance several times, such as learning their breasts, tracking breasts, etc. For example, 8 users stated that the BSE apps guide them to follow BSE moves. Also, 4 participants expressed their satisfaction in using the apps, which make them perform BSE regularly. Finally, the participants stated the importance of Support related aspects of apps several times, such as, feeling responsible for their health and feeling less anxious, etc.

To sum up, it was identified numerous categories and sub-categories under six heading based on women's behaviour, motivations, concerns, preferences, and expectations regarding BSE and the usage of mobile BSE apps. For example, it is found that the utilization of mobile BSE apps had some positive effects on the BSE experience, like performing BSE more attentively. In the light of participants' statements, it is believed that mobile BSE apps that adopt a user centered approach and were designed based on the needs and expectations of women would provide women a positive BSE experience. Consequently, these categories and sub-categories were defined as design strategies that provide insight for a positive mobile BSE app experience.

\section{POSITIVE TECHNOLOGY AND ITS RELEVANCE FOR THE BSE APPS}

The fieldwork helped to understand about women's BSE experiences and highlighted the importance of positive BSE experience. This gave insights into what design strategies should be followed for mobile BSE apps to contribute to women's healthcare and happiness. The design strategies for BSE apps based on the fieldwork results now will be discussed with their relevance to Positive technology.

\subsection{Hedonic Level of Positive Technology}

As briefly mentioned in the literature review, the hedonic level aims to develop positive emotions such as pleasure, relaxation, and enthusiasm by using technology (Botella et al., 2012). As a result of the field study that aims to determine design strategies for positive experiences with the use of mobile BSE apps, it was seen that the usage of the apps drove some positive emotions, which is one of the positive technology levels. Example statements from the participants were: "it made me feel responsible for my health", "it made me feel safe", "it kept me positive", and "it made me believe I am not alone". According to the fieldwork results, some dimensions that help to create experiences related to the hedonic level include: providing an uplifting experience, being humorous, and creating selfawareness.

\subsection{Eudaimonic Level of Positive Technology}

Eudaimonic level concentrates on technologies that support people to engage and self-actualize (Riva and Gaggioli, 2015). According to the participants' statements during the pre-usage phase, women had little knowledge of BSE and had concerns about a disease and finding a symptom; however, they were still eager to learn and perform BSE. Generally, as women performed BSE and obtained information about themselves, their enthusiasm to use the mobile BSE apps decreased. In order to create a comprehensive experience and keep users engaged, necessary dimensions that a BSE app should have include: being informative, being multifunctional, providing daily suggestions, allowing personalization, and encouraging active involvement. 


\subsection{Social-Interpersonal Level of Positive Technology}

During the analysis, it was found out that some of the BSE app features provide a sense of community feel, which is related to one of the positive technology levels. Social/Interpersonal level interested in technologies that are supporting and improving the commitment of individuals, communities, and groups among themselves. The majority of the participants suggested that features that allow users to socialize with friends or with other users could be advantageous for continuing to use the apps. Moreover, the participants mentioned that collaboration with doctors and healthcare clinics could improve their experience. Some dimensions that support participants' experiences related to socialinterpersonal level included being collaborative, and allowing social networking.

\section{CONCLUSION}

The fieldwork reveals the users' preferences, thoughts and expectations about the BSE apps. The results reveal six potential categories that design strategies for mobile BSE apps can be directed at: strategies, limitations, emotions under BSE theme, and needs, limitations, and benefits under Mobile Apps theme. The emerged categories as design strategies were interpreted through three levels of positive technology (i.e., hedonic, eudaimonic, and social \& interpersonal levels), to propose design dimensions that can be used to design for positive experience regarding mobile BSE apps.

Although the scope was related to BSE and mobile BSE apps, results and findings can be a reference for a mobile app design that is more specific to women's health by taking advantage of participants' needs and expectations. The study also presents insights about mobile BSE apps in terms of their strengths and weaknesses, numerous factors that support positive experiences, and their relationships with positive technology and its elements.

The study involved participants with different BSE experience levels. Future studies can be conducted with more people with similar BSE experience levels as it can enrich the results and can help to identify more specific design strategies for people. The study was carried through the usage of three mobile BSE apps, including a wider range of apps may reveal diverse features for BSE apps. The results presented in this paper believed to be useful as they helped to understand women's current attitudes, fears and expectations whilst performing BSE; and showed that (with the help of pre- and post- usage PANAS results) using a BSE app indeed increases women's positive affect and decreases negative affect. This offers an insightful feedback for the potential of further developing BSE apps, and the results offer directions for what design strategies and positive technology approaches can be relevant to consider for the development of future BSE apps.

\section{REFERENCES}

Al-Azmy, S.F., Alkhabbaz, A., Almutawa, H.A., Ismaiel, A.E., Makboul, G. and El-Shazly, M.K., 2013. Practicing breast self-examination among women attending primary health care in Kuwait. Alexandria Journal of Medicine, 49(3), pp.281-286.

Bender, J.L., Yue, R.Y.K., To, M.J., Deacken, L. and Jadad, A.R., 2013. A lot of action, but not in the right direction: systematic review and content analysis of smartphone applications for the prevention, detection, and management of cancer. Journal of medical Internet research, 15(12), p.e287.

Bogdan, R. and Biklen, S.K., 1997. Qualitative research for education. Boston, MA: Allyn \& Bacon.

Bolier, L., \& Abello, K. M. (2014). State of the art and future directions. Wiley-Blackwell Handb. Posit. Psychol. Interv. Wiley Blackwell: Chichester, West Sussex, 286-309.

Botella, C., Riva, G., Gaggioli, A., Wiederhold, B.K., Alcaniz, M. and Banos, R.M., 2012. The present and future of positive technologies. Cyberpsychology, Behavior, and Social Networking, 15(2), pp.78-84.

Downey, L., 2015. Well-being technologies: Meditation using virtual worlds.

Fogg, B.J., 2009, April. A behavior model for persuasive design. In Proceedings of the 4th international Conference on Persuasive Technology (pp. 1-7).

Gaggioli, Andrea, Giuseppe Riva, Dorian Peters, and Rafael A. Calvo. "Positive technology, computing, and design: shaping a future in which technology promotes psychological well-being." In Emotions and affect in human factors and human-computer interaction, pp. 477-502. Academic Press, 2017.

Giunti, G., Giunta, D.H., Guisado-Fernandez, E., Bender, J.L. and Fernández-Luque, L., 2018. A biopsy of Breast Cancer mobile applications: state of the practice review. International journal of medical informatics, 110, pp.1-9.

Houghton, L.C., Howland, R.E. and McDonald, J.A., 2019. Mobilizing breast cancer prevention research through smartphone apps: a systematic review of the literature. Frontiers in public health, 7, p.298. 
IARC: International Agency for Research on Cancer (2020) Cancer Today. Retrieved from https://gco.iarc.fr/today/online-analysis-multi-bars

Kaptein, M., De Ruyter, B., Markopoulos, P. and Aarts, E., 2012. Adaptive persuasive systems: a study of tailored persuasive text messages to reduce snacking. ACM Transactions on Interactive Intelligent Systems (TiiS), 2(2), pp.1-25.

Kissal, A. and Beser, A., 2011. Knowledge, facilitators and perceived barriers for early detection of breast cancer among elderly Turkish women. Asian Pac J Cancer Prev, 12(4), pp.975-984.

Lilley, D., 2009. Design for sustainable behaviour: strategies and perceptions. Design Studies, 30(6), pp.704-720.

Lockton, D., Harrison, D. and Stanton, N.A., 2010. The Design with Intent Method: A design tool for influencing user behaviour. Applied ergonomics, 41(3), pp.382-392.

McDowell, I., 2006. Measuring health: a guide to rating scales and questionnaires. Oxford University Press, USA.

McKay, F.H., Cheng, C., Wright, A., Shill, J., Stephens, H. and Uccellini, M., 2018. Evaluating mobile phone applications for health behaviour change: a systematic review. Journal of telemedicine and telecare, 24(1), pp.22-30.

Misovich, S.J., Martinez, T., Fisher, J.D., Bryan, A. and Catapano, N., 2003. Predicting Breast SelfExamination: A Test of the Information-Motivation-Behavioral Skills Model 1. Journal of Applied Social Psychology, 33(4), pp.775-790.

Özkan, İ. and Taylan, S., 2020. Barriers to women's breast cancer screening behaviors in several countries: A meta-synthesis study. Health Care for Women International, pp.1-31.

Ribeiro, N., Moreira, L., Almeida, A.M. and Santos-Silva, F., 2016. Happy: cancer prevention using smartphones. Procedia Computer Science, 100, pp.466-473.

Ribeiro, N., Moreira, L., Almeida, A.M.P. and Santos-Silva, F., 2017. Pilot study of a smartphone-based intervention to promote cancer prevention behaviours. International journal of medical informatics, 108, pp.125-133.

Riva, G. and Gaggioli, A., 2015. 3 Positive Change and Positive Technology. In Enabling Positive Change (pp. 39-52). Sciendo Migration.

Watson, D., Clark, L.A. and Tellegen, A., 1988. Development and validation of brief measures of positive and negative affect: the PANAS scales. Journal of personality and social psychology, 54(6), p.1063.

Yang, R.J., Huang, L.H., Hsieh, Y.S., Chung, U.L., Huang, C.S. and Bih, H.D., 2010. Motivations and reasons for women attending a breast self-examination training program: a qualitative study. BMC women's health, 10(1), pp.1-11. 


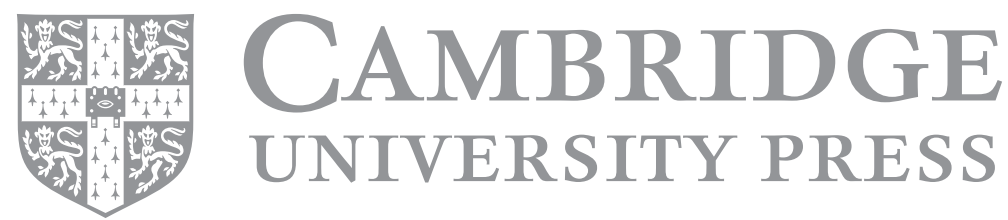

